\title{
Stimulus History Reliably Shapes Action Potential Waveforms of Cortical Neurons
}

\author{
Gonzalo G. de Polavieja, ${ }^{1,2 \star}$ Annette Harsch, ${ }^{1}$ Ingo Kleppe, ${ }^{1}$ Hugh P. C. Robinson, ${ }^{1}$ and Mikko Juusola ${ }^{1 \star}$ \\ ${ }^{1}$ Physiological Laboratory, University of Cambridge, Cambridge CB2 3EG, United Kingdom, and ${ }^{2}$ Neural Processing Laboratory, Department of Theoretical \\ Physics, Universidad Autónoma de Madrid, 28049 Madrid, Spain
}

Action potentials have been shown to shunt synaptic charge to a degree that depends on their waveform. In this way, they participate in synaptic integration, and thus in the probability of generating succeeding action potentials, in a shape-dependent way. Here we test whether the different action potential waveforms produced during dynamical stimulation in a single cortical neuron carry information about the conductance stimulus history. When pyramidal neurons in rat visual cortex were driven by a conductance stimulus that resembles natural synaptic input, somatic action potential waveforms showed a large variability that reliably signaled the history of the input for up to $50 \mathrm{~ms}$ before the spike. The correlation between stimulus history and action potential waveforms had low noise, resulting in information rates that were three to four times larger than for the instantaneous spike rate. The reliable correlation between stimulus history and spike waveforms then acts as a local encoding at the single-cell level. It also directly affects neuronal communication as different waveforms influence the production of succeeding spikes via differential shunting of synaptic charge. Modeling was used to show that slow conductances can implement memory of the stimulus history in cortical neurons, encoding this information in the spike shape.

Key words: action potential; AMPA; conductance; cortex; noise; information; synaptic communication; NMDA; GABA

\section{Introduction}

Action potentials are known to shunt incoming EPSPs (Coombs et al., 1955). Simple neuron models such as integrate-and-fire neurons assume that this shunting completely resets the membrane for synaptic integration (Koch, 1999). Recently, experiments on pyramidal and Purkinje neurons have demonstrated that the shunting is not in general complete (Häusser et al., 2001). The amount of charge that survives each action potential depends on the shape of both EPSP and action potential and on the relative timing between them. In this work, however, single backpropagating action potentials of a constant shape in each trial were used, and all underlying conductances returned to their resting values between trials. An influence of action potential shape was evident only from differences between pyramidal and Purkinje neurons and from modeling. A possible mechanism for variability in the action potential shape within a neuron is the

\footnotetext{
Received Jan. 18, 2005; revised May 5, 2005; accepted May 6, 2005.

This work was supported by the "Ramón y Cajal" program (G.G.d.P.), the Fundacion Banco de Bilbao Vizcaya Argentaria (G.G.d.P.), the Ministerio de Ciencia y Tecnología (G.G.d.P.), the Royal Society (M.J.), the Biotechnology and Biological Sciences Research Council (M.J., H.P.C.R.), the Gatsby Charitable Foundation (M.J.), the European Community (H.P.C.R.), and the Wellcome Trust (G.G.d.P., M.J.). We thank Andrew French for comments on a previous version of this manuscript and Michael Berry, William Bialek, Dmitri Chklovskii, Simon Laughlin, and Samue Wang for discussions.

*G.G.d.P. and M.J. contributed equally to this work.

Correspondence should be addressed to either of the following: Gonzalo G. de Polavieja, Neural Processing Laboratory, Department of Theoretical Physics, Universidad Autónoma de Madrid, 28049 Madrid, Spain, E-mail: gonzalo.polavieja@uam.es; or Mikko Juusola, Physiological Laboratory, University of Cambridge, Cambridge CB2 3EG, UK, E-mail: mj216@cam.ac.uk.

DOI:10.1523/JNEUROSCI.0242-05.2005

Copyright $\odot 2005$ Society for Neuroscience $\quad$ 0270-6474/05/255657-09\$15.00/0
}

variable participation of dendritic sodium currents, which can be prevented by proximal IPSPs (Paré et al., 1998).

An extra source of variability in action potential waveforms in vivo is attributable to activity. Even injecting a steady current step produces several action potentials of different shapes, with a correlation between spike frequency and spike broadening (Kandel and Spencer, 1961; Aldrich et al., 1979; Fox and Ranck, 1981). Sodium and potassium channels are responsible for activitydependent spike amplitude reduction and broadening (Grossman et al., 1979; Coates and Bullock, 1985; Gillette et al., 1980; Jackson et al., 1991; Wang and Kaczmarek, 1998; Geiger and Jonas, 2000).

Such experiments are valuable for exposing the underlying mechanisms, but to understand the effect of stimulus history, one needs a naturalistic, dynamically rich stimulation and a probabilistic analysis. In this study, we use dynamic conductance stimulus patterns to investigate whether spike shapes reliably depend on previous stimulus history. We show that, in cortical neurons, action potential waveforms depend on the previous $50 \mathrm{~ms}$ of conductance stimulus history. This relationship is low in noise, carrying three to four times more information than the spike times only. In contrast to spike broadening during simple current steps, in which spike frequency and waveform are directly related, spike frequency is a poor encoder of conductance history in our experiments. Although spike frequency and spike shape are related on average, it is only spike shape that depends reliably on different features of the stimulus history on a trial-by-trial basis. We show by modeling our experiments that slow conductances can act as a memory of the conductance history and serve to encode it in the spike shape. 
Because cortical action potentials of different waveforms differentially shunt incoming synaptic events, they participate in the synaptic integration and the generation of succeeding action potentials (Häusser et al., 2001). Therefore, at least $50 \mathrm{~ms}$ of the stimulus history can affect the state of synaptic integration of the neuron and the generation of spikes, through modulation of the action potential waveform. This is a form of local encoding at the single-neuron level with a 50-ms-long memory, which affects neuronal communication by influencing the production of future spikes.

\section{Materials and Methods}

Preparation and electrophysiology. In vitro single-electrode whole-cell recordings from the somas of neurons in cortical layers $2 / 3$ and 5 were performed in transverse slices prepared from occipital cortex of 13- to 23-d-old Wistar rats. A Multiclamp 700A amplifier (Axon Instruments, Union City, CA) was used in a fast "bridge" current-clamp mode, with patch pipettes having resistances of $4-6 \mathrm{M} \Omega$. Slices were viewed with an upright microscope (BW50WI; Olympus Optical, Tokyo, Japan) by infrared differential interference contrast optics. Signals were filtered at 5 $\mathrm{kHz}(-3 \mathrm{~dB}$, four-pole Bessel) and sampled with 12-bit resolution at 20 $\mathrm{kHz}$. During recording, the slices were perfused continuously with Ringer's solution (in mM): $125 \mathrm{NaCl}, 2.5 \mathrm{KCl}, 25 \mathrm{NaHCO}_{3}, 25$ glucose, 1.25 $\mathrm{NaH}_{2} \mathrm{PO}_{4}, 2 \mathrm{CaCl}_{2}$, and $1 \mathrm{MgCl}_{2}$. The Ringer's solution was equilibrated with $95 \% \mathrm{O}_{2}, 5 \% \mathrm{CO}_{2}$ gas to a final $\mathrm{pH}$ of 7.4. Additionally, $10 \mu \mathrm{M}$ bicuculline, $10 \mu \mathrm{M}$ CNQX, and $10 \mu \mathrm{M}$ APV (Tocris Cookson, Ballwin, $\mathrm{MO}$ ) were included to block intrinsic synaptic conductances. The pipette solution (Harsch and Robinson, 2000) contained $20 \mathrm{~mm}$ phosphocreatine $\mathrm{Na}_{2}, 4 \mathrm{mM} \mathrm{MgCl}_{2}, 0.3 \mathrm{~mm}$ GTP, $4 \mathrm{~mm}$ ATP, $100 \mathrm{~mm}$ potassium gluconate, $20 \mathrm{~mm} \mathrm{KCl}, 10 \mathrm{~mm} \mathrm{HEPES}$, and $5 \mathrm{U} / \mathrm{ml}$ creatine phosphokinase. It was balanced to $\mathrm{pH} 7.3$ with $\mathrm{NaOH}$. All experiments were performed either at room temperature $\left(21-23.5^{\circ} \mathrm{C} ; n=32\right)$ or a nearphysiological temperature $\left(34 \pm 0.5^{\circ} \mathrm{C} ; n=24\right)$.

Conductance injection. Cells were stimulated using the conductance injection technique, also known as dynamic clamp (Robinson, 1991; Robinson and Kawai, 1993; Sharp et al., 1993), which, unlike currentclamp stimulation, allows a natural interaction between input current and the membrane potential (for a comparison of conductance and current stimulation, see Supplement 1, available at www.jneurosci.org as supplemental material). The opening of AMPA, NMDA, and GABA receptors was modeled by injecting a current $I(t)$ that depended instantaneously on the changing membrane potential $V(t)$ (in $\mathrm{mV}$ ):

$$
\begin{aligned}
I(t)=g_{\mathrm{AMPA}}(t)\left[V(t)-E_{\mathrm{AMPA}}\right]+g_{\mathrm{GABA}}(t)\left[V(t)-E_{\mathrm{GABA}}\right] & \\
& +\frac{g_{\mathrm{NMDA}}(t)}{1+k_{1} \exp \left(-k_{2} V(t)\right)}\left[V(t)-E_{\mathrm{NMDA}}\right],
\end{aligned}
$$

with $E_{\mathrm{AMPA}}=E_{\mathrm{NMDA}}=0 \mathrm{mV}$, and $E_{\mathrm{GABA}}$ equal to the resting potential $\left(-65.0 \pm 5.1 \mathrm{mV} ;\right.$ mean $\left.\pm \mathrm{SD} ; n=24 ; t=34^{\circ} \mathrm{C}\right)$. The constants $k_{1}=0.6$ and $k_{2}=0.06$ describe the voltage-dependent magnesium block of the NMDA receptors. The AMPA, GABA, and NMDA conductances were each a sum of elementary synaptic events, $\mathrm{g}(\mathrm{t})=\Sigma_{\mathrm{t}} \mathrm{g}_{\mathrm{i}}(\mathrm{t})$, each described by $\lambda(t)=\bar{R} \sum_{i} \exp \left(-\left(t-T_{i}\right) / \tau_{\delta}\right) g i(t)=\bar{g}\left[\exp \left(-\left(t-t_{i}\right) / \tau_{d}-\right.\right.$ $\exp \left(-\left(t-t_{i}\right) \tau_{r}\right]$ for $t \leq t_{i}$ and zero for $t \leq t_{i}$. The scaling constant $\bar{g}=$ 1000,100 , and $300 \mathrm{pS}$, the decay time constant $\tau_{d}=2,150$, and $7 \mathrm{~ms}$, and the rise time constant $\tau_{r}=0.5,5$ and $0.5 \mathrm{~ms}$ for AMPA, NMDA, and GABA, respectively. The initiation times for the unitary events, AMPA/ NMDA, or GABA were each determined by a Poisson process, with time intervals $T$ between events distributed as $p(T)=\lambda \exp (-\lambda T)$ or from a nonstationary Poisson process whose rate is given by for $t \geq T_{i}$, with $\bar{R}$ the initial peak rate, $\tau_{b}$ the burst decay time constant, and $\left\{T_{i}\right\}$ the burst times drawn from a Poisson distribution. $\bar{R}$ and $\tau_{b}$ were adjusted individually for each neuron to evoke vigorous firing. The command signal for conductance injection was generated using an SM-1 conductance injection amplifier (Cambridge Conductance, Cambridge, UK). Membrane potential was not corrected for liquid junction potential.

We recorded voltage responses of neurons to either a continuously generated stochastic conductance pattern or a conductance pattern that
Table 1. Action potential parameters at room temperature

\begin{tabular}{lllll}
\hline 22-23.5 $5^{\circ} \mathrm{C}$ & Parameter & Mean & SD & $n$ \\
\hline All neurons & Firing rate & $5.2 \mathrm{spikes} / \mathrm{s}$ & $2.4 \mathrm{spikes} / \mathrm{s}$ & 32 \\
& ISI (peak of pdf) & $69.6 \mathrm{~ms}$ & $25.6 \mathrm{~ms}$ & 32 \\
& Height/width gradient & $1.5 \mathrm{mV} / \mathrm{ms}$ & $0.7 \mathrm{mV} / \mathrm{ms}$ & 32 \\
Layer 5 & Firing rate & $6.1 \mathrm{spikes} / \mathrm{s}$ & $2.7 \mathrm{spikes} / \mathrm{s}$ & 16 \\
& ISI (peak of pdf) & $65.2 \mathrm{~ms}$ & $25.5 \mathrm{~ms}$ & 16 \\
& Height/width gradient & $1.2 \mathrm{mV} / \mathrm{ms}$ & $0.6 \mathrm{mV} / \mathrm{ms}$ & 16 \\
Layer 2/3 & Firing rate & $4.3 \mathrm{spikes} / \mathrm{s}$ & $1.8 \mathrm{spikes} / \mathrm{s}$ & 13 \\
& ISI (peak of pdf) & $74.0 \mathrm{~ms}$ & $24.6 \mathrm{~ms}$ & 13 \\
& Height/width gradient & $1.8 \mathrm{mV} / \mathrm{ms}$ & $0.6 \mathrm{mV} / \mathrm{ms}$ & 13 \\
\hline
\end{tabular}

pdf, Probability density function.

was repeated $80-200$ times, with each presentation lasting 6-20 s. In either case, the total duration of the recordings was 10-40 min. The results described in this paper hold true for both of these stimuli. Neurons were considered for analysis only if their membrane potential and their responses showed no obvious drifts; the data were rejected if the resting potential or the number of action potentials varied more than $10 \%$ across the traces. The total number of action potentials in a single experiment varied from $\sim 1000$ to $\sim 20,000$. Data acquisition was performed by a purpose-built Matlab interface (MathWorks, Natick, MA)
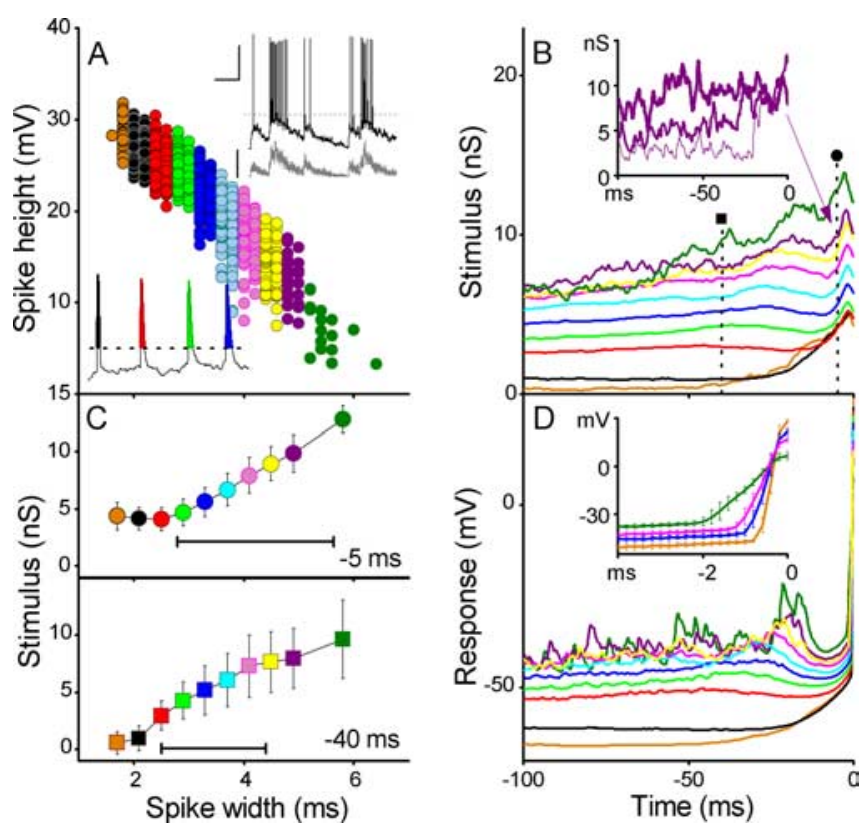

Figure 1. Action potential waveforms of pyramidal neurons encode information about the stimulus history. $\boldsymbol{A}$, The heights and widths of action potentials (filled circles; $n=4137$ ) occupy a large linear range during naturalistic conductance stimulation; here, height $=37.4-4.8 \times$ width. This recording is from cortical layer $2 / 3\left(13-d-o l d\right.$ rat at $\left.34^{\circ} \mathrm{C}\right)$. Action potential waveforms (top inset; black trace) vary with synaptic input patterns of a sum of AMPA unitary events (top inset; gray trace). Calibration: $30 \mathrm{mV}, 0.5 \mathrm{~s}$. Calibration bar for conductance: $10 \mathrm{nS}$. The thin horizontal line indicates the voltage $(-30 \mathrm{mV})$ at which the action potential widths were measured. The widths are color coded into 10 groups (bottom inset, 9 equally spaced with the 10th, including the remainder), from narrow to wide as follows: orange, black, red, green, cyan, blue, magenta, yellow, purple, and olive. $\boldsymbol{B}$, Average stimulus (conductance) history preceding the peak of the action potentials for each group (same coloring). The black circle and square indicate the conductance values at 5 and $40 \mathrm{~ms}$ before the spiking time, respectively. The inset shows three different conductance histories (thick, medium, and thin traces) leading to a similar spike (purple). C, Mean and SD of the conductance histories for each action potential group at 5 $\mathrm{ms}$ (top window) and $40 \mathrm{~ms}$ (bottom window) before firing. Horizontal bars indicate where the means of the conductance histories of the neighboring groups differ significantly by one-way ANOVA ( $p<10-8$ ). D, Average voltage responses preceding the action potential waveforms (same grouping and coloring). The inset shows the mean and SD of the voltage response $4 \mathrm{~ms}$ before the spike peak for four action potential groups. 

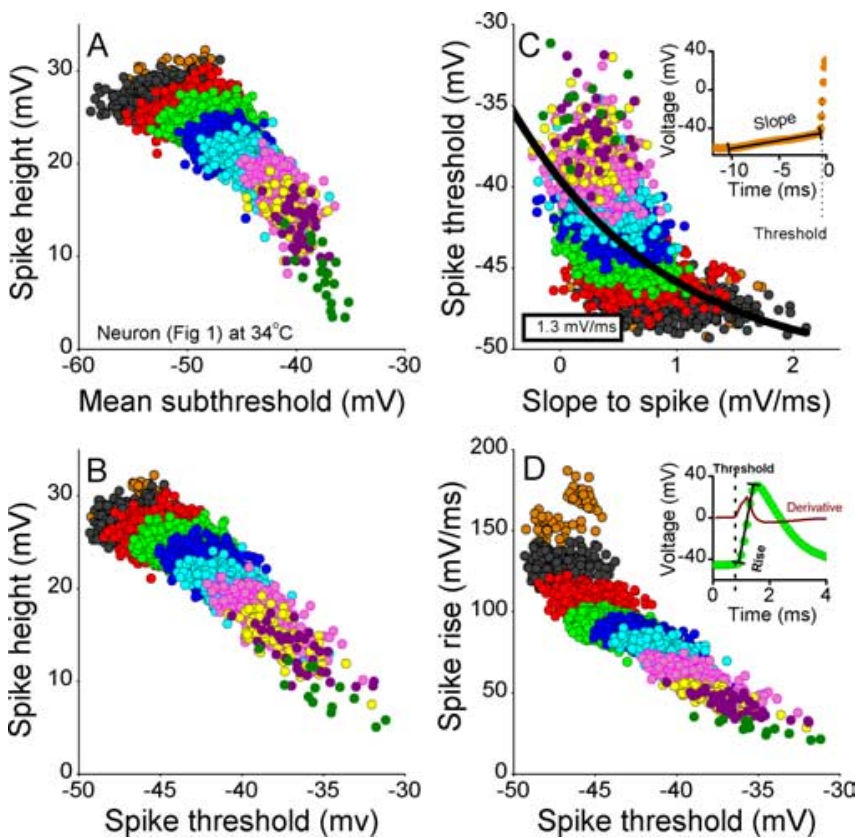

Figure 2. Analysis of the voltage history. $\boldsymbol{A}$, Spike height versus mean subthreshold voltage calculated for the $10 \mathrm{~ms}$ preceding each action potential ( filled circles; $n=4137$; same data and color coding as in Fig. 1). $\boldsymbol{B}$, Spike height versus spike threshold calculated for each action potential. C, Spike threshold shows an exponential relationship with the preceding voltage slope, with a decay constant of 1.3. D. The rising slope of the spike (spike rise) also correlates strongly with the threshold. The inset shows how the spike rise was calculated.

Table 2. Action potential parameters at near-physiological temperature

\begin{tabular}{lllll}
\hline $34 \pm 0.5^{\circ} \mathrm{C}$ & Parameter & Mean & SD & $n$ \\
\hline All neurons & Firing rate & 7.3 spikes $/ \mathrm{s}$ & $3.5 \mathrm{spikes} / \mathrm{s}$ & 24 \\
& ISI (peak of pdf) & $38.9 \mathrm{~ms}$ & $28.9 \mathrm{~ms}$ & 24 \\
& Height/width gradient & $2.4 \mathrm{mV} / \mathrm{ms}$ & $1.0 \mathrm{mV} / \mathrm{ms}$ & 24 \\
Layer 5 & Firing rate & $6.4 \mathrm{spikes} / \mathrm{s}$ & $4.5 \mathrm{spikes} / \mathrm{s}$ & 13 \\
& ISI (peak of pdf) & $27.2 \mathrm{~ms}$ & $0.7 \mathrm{~ms}$ & 13 \\
& Height/width gradient & $1.8 \mathrm{mV} / \mathrm{ms}$ & $1.0 \mathrm{mV} / \mathrm{ms}$ & 13 \\
Layer 2/3 & Firing rate & $8.5 \mathrm{spikes} / \mathrm{s}$ & $1.8 \mathrm{spikes} / \mathrm{s}$ & 8 \\
& ISI (peak of pdf) & $33.3 \mathrm{~ms}$ & $18.4 \mathrm{~ms}$ & 8 \\
& Height/width gradient & $3.1 \mathrm{mV} / \mathrm{ms}$ & $1.0 \mathrm{mV} / \mathrm{ms}$ & 8 \\
\hline
\end{tabular}

pdf, Probability density function.

with an interface package for National Instruments (Austin, TX) boards (MATDAQ; H. P. C. Robinson, 1997-2005).

Conductance histories preceding action potentials of given widths. The widths of action potentials were measured at a given voltage level $(-20$ to $-40 \mathrm{mV}$ ) set so as to exclude subthreshold membrane potentials and were sorted into 10 groups. Results were independent of the voltage level chosen for width measurements and of the number of groups for analysis. The conductance histories preceding the action potentials of each width group were used to calculate the corresponding mean conductance histories and SDs. All neurons tested showed results similar to those in Figures 1 and 2.

Conductance histories preceding interspike intervals. The interspike intervals (ISIs) were measured from the peak of each action potential to the peak of the next one. Because the mean interspike interval in the recordings was $55.5 \pm 29.8 \mathrm{~ms}(n=56)$, the ISIs were divided into nine groups around this value, using $10 \mathrm{~ms}$ intervals with the 10th group containing ISIs longer than $101 \mathrm{~ms}$ (see Fig. 4). Notice that, for bursty conductance stimulation, the ISI distributions have long tails (the 10th group) and a low mean spike rate (Table 1). There were at least 50 conductance histories in each ISI group. For all cells studied, the means and SDs of the conductance histories of the ISI groups behaved similarly to the data in Figure 5.

Wilson model of cortical neuron. Mammalian cortical neurons have at least 12 different major ionic currents. Simplified models of their behav-
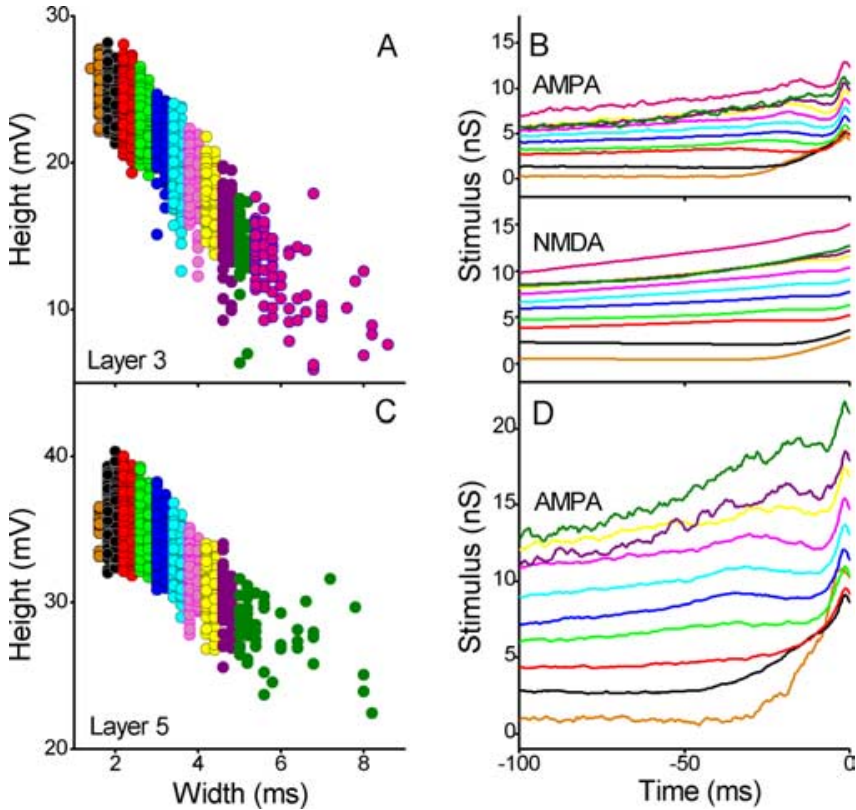

Figure 3. Waveform encoding is independent of cortical layer and different mixtures of conductances. $\boldsymbol{A}$, Height versus width for action potentials (filled circles; $n=4977$ ) from cortical layer $2 / 3$ (13-d-old rat at $34^{\circ} \mathrm{C}$ ). Heights and widths obey an approximately linear relationship (height $=30.4-2.9 \times$ width) during naturalistic input patterns made of AMPA and NMDA unitary events instead of AMPA as in Figure 1. The widths are color coded into 11 groups as follows: orange, black, red, green, cyan, blue, magenta, yellow, purple, olive, and royal. $\boldsymbol{B}$, Average AMPA and NMDA command conductance histories until the peak of the action potential for each group (same coloring). C, Height versus width for action potentials (filled circles; $n=$ 5081) from cortical layer $5\left(13-d\right.$-old rat at $\left.34^{\circ} \mathrm{C}\right)$ obey an approximately linear relationship (height $=39.1-2.1 \times$ width) during naturalistic input patterns made of AMPA unitary events only. The widths are color coded as before for 10 groups. D, Average AMPA conductance history until the peak of the action potential for each group in C (same coloring).

ior have been proposed by Wilson (1999a,b) that reproduce well the spike shape in response to a current step. The following Wilson model takes into account the $\mathrm{Na}^{+}$and $\mathrm{K}^{+}$spike-producing currents, the $\mathrm{Ca}^{2+}$ current $I(t)$, and hyperpolarizing current $I_{\mathrm{AHP}}$. The Wilson model is based on an equation for the voltage change $C d V / d t=-m_{\infty}(V-0.5)-$ $26 R(V+0.95)-0.5 T(V-1.2)-2.5 H(V+0.95)+I_{\text {ext }}$ and the equations $d R / d t=\left(-R+R_{\infty}\right) / 4.2, d T / d t=\left(-T+T_{\infty}\right) / 14$, and $d H / d t=$ $(-H+3 T) / 120$ with $m_{\infty}=17.8+47.6 V+33.8 V^{2}, T_{\infty}=8(V+0.725)^{2}$, and $R_{\infty}=0.79+1.29 \mathrm{~V}+3.3(\mathrm{~V}+0.38)^{2}$, with voltage units in $\mathrm{mV} / 100$. $I_{\text {ext }}$ is given by Equation 1, thus modeling our conductance injection experiments using stochastically timed trains of unitary conductance transients. Results were robust under changes of parameters. We also considered models with four spatial compartments to study the effect of dendritic sodium channels (Supplement 3, available at www.jneurosci.org as supplemental material). The first three compartments have the coupling terms $4\left(V_{k-1}-V_{k}\right)+4\left(V_{k+1}-V_{k}\right)$ and the fourth compartment only the first of these two terms (Wilson, 1999b). Numerical integration of these equations was done with custom-built Matlab software and analyzed identically to the experimental data.

\section{Results}

Stimulus features preceding different spike waveforms

To examine the effect of stimulus history in the action potential waveforms, we performed whole-cell recordings from pyramidal neurons of rat cortex. To mimic natural synaptic input, we used the technique of conductance injection, or dynamic clamp (Robinson and Kawai, 1993; Sharp et al., 1993; Harsch and Robinson, 2000). Our stimulus consisted of stochastically timed trains of unitary conductance transients with the characteristics of glutamatergic synaptic inputs (see Materials and Methods). Figure $1 \mathrm{~A}$ 

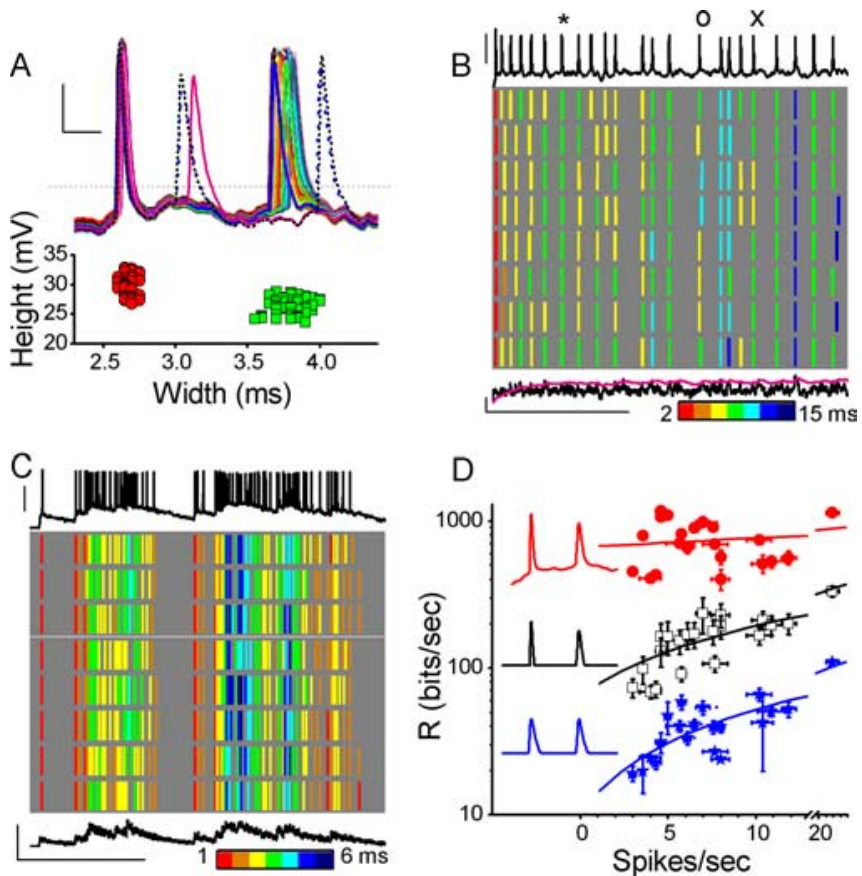

Figure 4. Reliable action potential waveforms in repetition experiments and high information transfer rate. $\boldsymbol{A}$, Two successive action potentials evoked 100 times by the same conductance stimulus pattern and their heights and widths (below) measured at $-35 \mathrm{mV}$ (thin dotted line). Calibration: $20 \mathrm{mV}, 10 \mathrm{~ms}$. $\boldsymbol{B}$, Top trace is an intracellular voltage response of a pyramidal neuron in layer $2 / 3$ (calibration, $40 \mathrm{mV}$ ) to a stationary conductance pattern of stochastically timed trains of AMPA and NMDA events (bottom traces, black and red lines, respectively; calibration: $5 \mathrm{nS}, 1 \mathrm{~s}$ ) at $22^{\circ} \mathrm{C}$ (see Materials and Methods). Between the action potentials are represented by bars with colors indicating the spike width ("temperature"-colored scale indicated at the bottom; red, thin and narrow spikes; blue, short and wide spikes). Rows correspond to consecutive responses to the same conductance pattern (8 traces). $C$, Voltage response (top trace; calibration, $40 \mathrm{mV}$ ) of neuron in layer 5 to a bursting conductance pattern of AMPA events (bottom trace; calibration: $5 \mathrm{nS}, 1 \mathrm{~s}$ ) at $34^{\circ} \mathrm{C}$ (see Materials and Methods). Between are shown the corresponding "temperature"-colored widths during eight stimulus trials. $\boldsymbol{D}$, Information transfer rate of pyramidal neurons ( $n=21$ ) for different conductance patterns (both stationary and nonstationary) plotted against the mean firing rate of each experiment. The information transfer rate was calculated for the full voltage responses (red filled circles), the action potential waveforms (white squares), and stereotyped action potentials (blue stars), each sampled at 2 $\mathrm{kHz}$. The inset shows a short sample of the three waveforms containing two action potentials. Information transfer rate, $R$, for each signal type increases with the mean firing rate, $f$ (full voltage responses: $r=10.4 \times f+660.6$, red line; action potential waveforms: $r=12.9 \times$ $f+64.7$, black line; stereotyped spikes: $r=4.2 \times f+9.8$, blue line).

shows a typical recording and the heights and widths of the action potentials. The height is the peak absolute voltage value, and the width of an action potential is measured close to its base at a fixed voltage level (here at $-30 \mathrm{mV}$ ). There is a large variability in the waveforms of action potentials, with heights varying typically between 0 and $30 \mathrm{mV}$ and widths between 1 and $6 \mathrm{~ms}$. This variability, which was present in all of our recordings $(n=56)$, resembles that in published records of cortical neuron firing during synaptic input in vivo (Carandini and Ferster, 2000) and is larger than during stimulation by current steps (Harsch and Robinson, 2000). Height and width were linearly correlated, ranging from tall and narrow to short and wide. Is this variability random attributable to the underlying stochastic dynamics of channels or does the action potential waveform carry information about particular changes in the stimulation?

To search for particular features that might be encoded by the waveform variability, we calculated the average stimulus preceding action potentials of given height and width ranges. Action potentials were sorted by width into 10 groups for analysis, indi- cated by different colors in Figure $1 A$. Figure $1 B$ shows the average conductance stimulus preceding a spike for each of these 10 groups using the same color scheme. Common to all groups is a fast conductance increase followed by a decrease within $5 \mathrm{~ms}$ before a spike. Each group, however, encodes a different level of stimulus amplitude. The broader and the shorter the action potential, the larger the stimulus amplitude it encodes. Another characteristic of this encoding is that, for the lowest overall conductance histories, there is a high rate of increase during the $10-20 \mathrm{~ms}$ before spike production. The same ordering of average stimulus levels for the different groups is seen $5 \mathrm{~ms}$ before spike production as for $100 \mathrm{~ms}$ earlier. To find the relevant time interval for encoding, we calculated the SD of stimulus histories. Figure $1 C$ (top) shows that, at $5 \mathrm{~ms}$ before spike production, the SDs are small enough to distinguish different conductance histories. As seen in the larger SDs at $40 \mathrm{~ms}$ before spikes (bottom), this specificity gradually deteriorates for times more distant from spike production. An example of individual conductance histories leading to the production of similar spikes is given in the inset of Figure $1 B$. Even very different input patterns, which converge to similar trajectories within the $20 \mathrm{~ms}$ before firing, lead to the same action potential waveform group. Figure $1 D$, which depicts the average voltage trajectories preceding action potentials in the different waveform groups, shows that each spike waveform class was produced at a different mean potential level before the firing. The inset shows the mean voltage histories within $4 \mathrm{~ms}$ before the peak of the action potentials; the small non-overlapping SDs of the action potential groups indicate that their waveforms are clearly distinguishable.

To better understand the possible mechanisms responsible for the variation in spike shapes, Figure 2 gives a more detailed analysis on a trial-to-trial basis of the potential levels before firing. The spike shape depends on the availability of sodium channels, which depends on the voltage history, which in turn is obviously correlated to the conductance input history. Figure $2 \mathrm{~A}$ shows a correlation between the spike height and the subthreshold potential averaged over the $10 \mathrm{~ms}$ preceding each spike. Grouping by widths follows the same color code as in Figure 1. We also tested whether the spike shape depends on the voltage slope leading into action potentials. Faster depolarization may be expected to lower the threshold and produce full-blown spikes, whereas, when the voltage lingers close to threshold before spike initiation, inactivation should generate spikes of lower amplitude (Azouz and Gray, 2000). This mechanism predicts a correlation between spike height and the spike threshold and between the voltage slope preceding a spike and the spike threshold, as shown in Figure 2, $B$ and $C$, respectively. Therefore, both the absolute voltage level value and the slope are relevant parameters of the voltage history in the $10 \mathrm{~ms}$ preceding different spike shapes. At times preceding $10 \mathrm{~ms}$ before a spike, there is a decreasing relevance of the slope, especially for the wider spikes, as is clear from the average voltage histories in Figure $1 D$. We further tested whether this relationship can be partially explained by the availability of sodium channels. For this, we use the slope during the rising phase of the spike (spike rise) as a measure of sodium current: a lower value indicates an increase in sodium channel inactivation (Haag and Borst, 1996; Azouz and Gray, 2000). Figure 2D gives the spike rise versus spike threshold. According to this figure, the higher threshold of smaller and wider spikes corresponds to a lower spike rise, that is, to a higher degree of sodium channel inactivation.

In the 56 cells tested from different cortical layers (layers 3 and 5 ), using different types of conductances (AMPA, AMPA plus GABA, and AMPA plus NMDA), different ages (13-23 d), and 

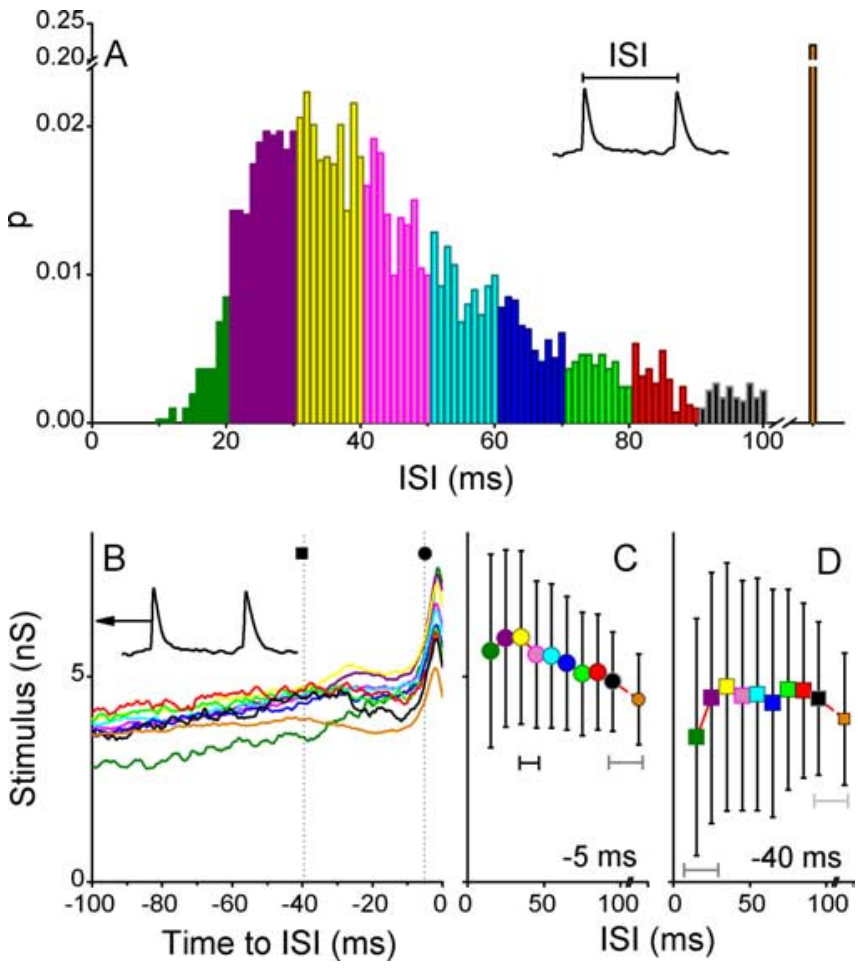

Figure 5. Low correlation between stimulus variations and instantaneous spike rate. $A$, The ISI probability density is shown for the same experiment as in Figure 1. ISIs are sorted into 10 groups: orange, 91-100 ms; black, 81-90 ms; red, 71-80 ms; green, 61-70 ms; blue, 51-60 ms; cyan, 41-50 ms; magenta, 31-40 ms; yellow, 21-30 ms; olive, 1-20 ms; black, >100 ms. $\boldsymbol{B}$, Mean conductance history before the ISIs of each group. The black circle and square indicate the conductance values at 5 and $40 \mathrm{~ms}$ before the ISIs, respectively. Mean conductance and SD (error bars) are shown for $5 \mathrm{~ms}(\boldsymbol{C})$ and $40 \mathrm{~ms}(\boldsymbol{D})$ before the ISIs. Horizontal bars indicate where the means of the conductance histories of the neighboring groups differ significantly by oneway ANOVA (black bar, $p<0.0005$; gray bar, $p<0.005$; light gray bar, $p<0.05$ ).

different temperatures $\left(23\right.$ or $\left.34^{\circ} \mathrm{C}\right)$, we found differences in the action potential shapes but always the same encoding principles. As an index of the correlation between height and width, Tables 1 and 2 show the gradient of the linear fits to the height/width distributions, in layers $2 / 3$ and 5 , at temperatures of 23 or $34^{\circ} \mathrm{C}$, respectively.

Figure 3 gives two examples that show analogous encoding properties in different layers and with different mixtures of conductances. Figure 3, $A$ and $B$, is similar to Figure 1 but for a stimulus made of AMPA and NMDA conductances. We show separately the AMPA and NMDA stimulus features producing action potentials of different widths. Wider potentials are produced for higher AMPA and NMDA conductances. Figure 3, $C$ and $D$, shows the waveform encoding for a layer 5 neuron. Action potentials have shapes different from those of layer 2/3 neurons, typically with half the height/width ratio. The AMPA stimulus features encoded are, however, indistinguishable from those of layer $2 / 3$ neurons.

\section{Reliability of encoding of stimulus history into spike waveforms}

To study quantitatively the reliability of the encoding into action potential waveforms, we performed experiments in which the same naturalistic input was applied repeatedly. Figure $4 \mathrm{~A}$ shows an example of 100 superimposed sections of these responses, in which two action potentials were reliably elicited. The heights and widths of the two spikes are shown below as two clusters. Both sets of action potentials conserved their shapes across trials.
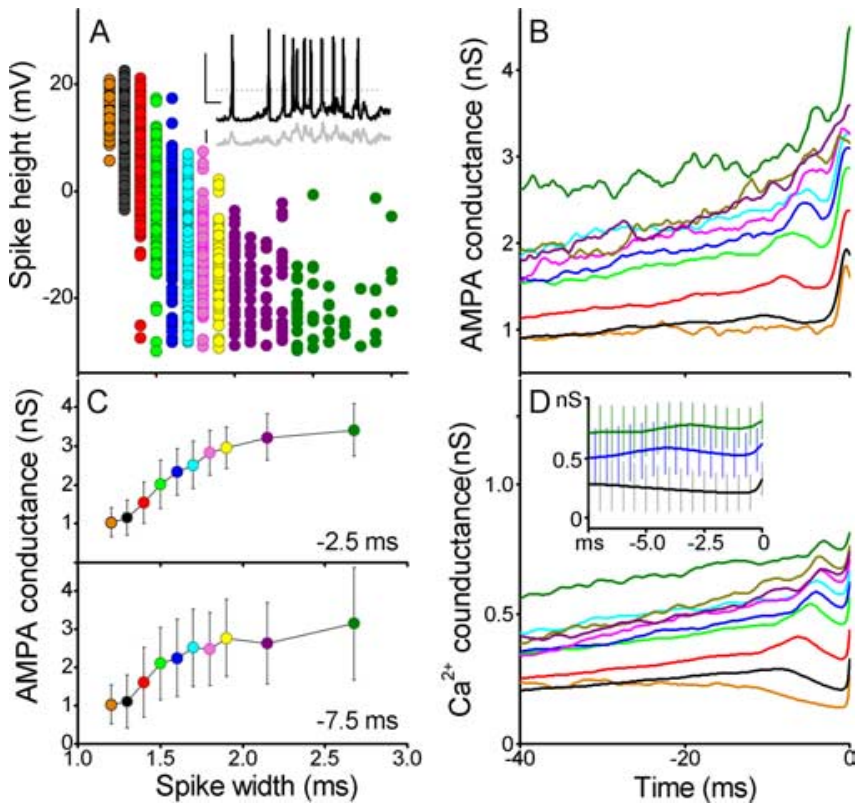

Figure 6. Action potential waveforms of a cortical neuron model dynamically encode information about the stimulus history. $\boldsymbol{A}$, The heights and widths of action potentials. Action potential waveforms (top inset; black trace) vary with synaptic input patterns of a sum of AMPA unitary events (top inset; gray trace). Calibration: $50 \mathrm{mV}, 20 \mathrm{~ms}$; calibration bar for conductance, $2 \mathrm{nS}$. The thin horizontal line indicates the voltage $(-42 \mathrm{mV})$ where the action potential widths were measured. The widths are color coded into 10 groups for analysis. $\boldsymbol{B}$, Average stimulus (conductance) history preceding the peak of the action potentials for each group (same coloring). $C$, Mean and SD of the conductance histories for each action potential group at $1.5 \mathrm{~ms}$ (top window) and $7.5 \mathrm{~ms}$ (bottom window) before firing. $\boldsymbol{D}$, Calcium conductance history before firing for each of the 10 spike waveform groups. The inset shows mean and SD for three groups.

Additional low-probability action potentials also preserved their shapes across trials. Figure $4, B$ and $C$, shows the first eight repetitions of responses to stationary and nonstationary (bursting) Poisson input trains, lasting 2.5 and $1.7 \mathrm{~s}$, respectively. Action potentials are color coded in a gradient from red (tall, thin) to blue (short, wide). This demonstrates that, during both types of stimulation, there was not only a high reliability of action potential times (Mainen and Sejnowski, 1995; Harsch and Robinson, 2000) but also of the action potential shapes throughout the response. Three representative cases in Figure $4 B$ are indicated by the symbols ${ }^{*}, \bigcirc$, and $\times$. At ${ }^{*}$, the action potentials were produced at the same time and all belonged to the same waveform group. At $\bigcirc$, there is a small spread in the spike times within the ensemble, but spikes at exactly the same times have the same widths. Although all spike times were similar at $\times$, differences in waveform stem from distinct preceding response histories.

The small SDs in the stimulus features encoded, shown in Figure $1 C$, and the repeatability of the action potential times and shapes in Figure 4, $B$ and $C$, show that there is a reliable encoding of $50 \mathrm{~ms}$ of the conductance stimulus into spike waveforms. It is desirable, however, to obtain a quantitative measure of this reliability that is independent of the features encoded, the particular shapes of the action potentials, or any underlying mechanisms. We used repeated trial experiments to calculate the rate of information transfer between input and output, a standard measure used previously for spike rates (MacKay and McCulloch, 1952; Barlow, 1961; de Polavieja, 2002), for spike times (Juusola and French, 1997; Strong et al., 1998; Reinagel and Reid, 2000), and for the responses of graded neurons (van Steveninck and Laughlin, 1996; Juusola and de Polavieja, 2003). We calculated the rate of information transfer for three different cases: the full voltage 

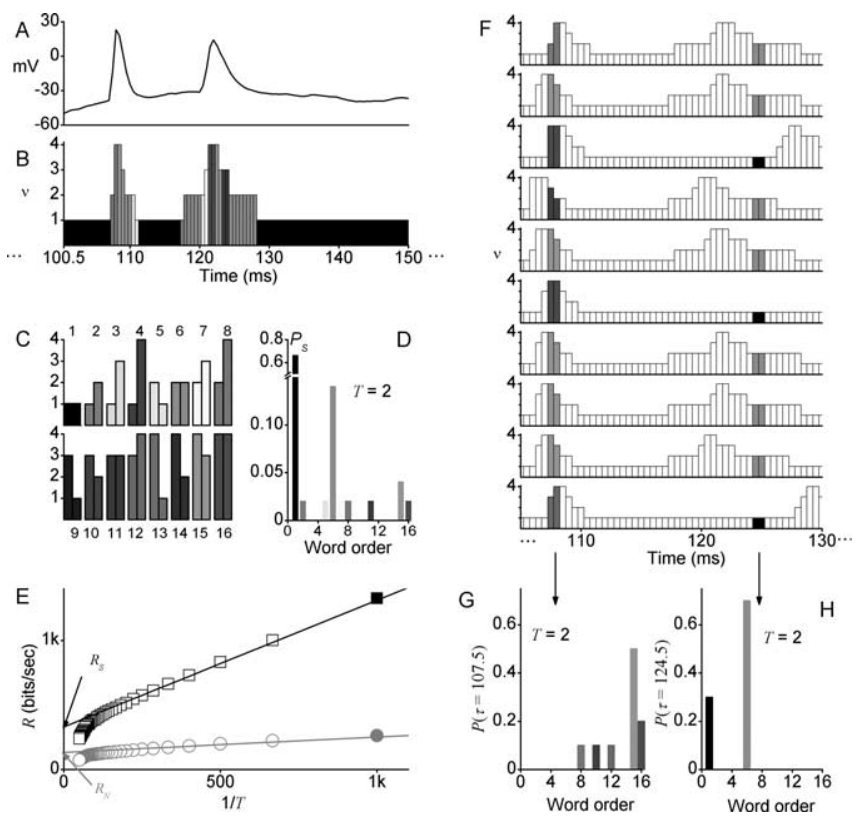

Figure 7. Calculation of the rate of information transfer from the digitized voltage response. $\boldsymbol{A}$, Voltage response in a $50 \mathrm{~ms}$ window. $\boldsymbol{B}$, Digitized version of the voltage response, shown here with four voltage levels. C, Sixteen possible "words" of length $T=2$ letters $=0.001 \mathrm{~s}$ and $v=$ 4 voltage levels. $D$, Probability of finding the 16 possible words in the digitized voltage response in $\boldsymbol{B}$. $\boldsymbol{E}$, Total and noise entropy rates, $R_{S}$ and $R_{N}$ in Equation 4 , obtained as the infinite length limit, $T \rightarrow \infty$, from the finite length values. Black squares indicate the values of the information rates before the infinite length limit for words of length $T=2$ letters $=0.001 \mathrm{~s}$ using the probability density in $\boldsymbol{D}$. $\boldsymbol{F}$, Digitized version of the voltage response for 12 repetitions in experiments with $80-200$ repetitions, shown for a time window of $15 \mathrm{~ms}$ and $v=4$ voltage levels. $\mathbf{G}$, Probability of finding the 16 two-letter words with four voltage levels at two different times in a trial and calculated across trials. These probabilities are used in the calculation of the noise rate, $R_{N}$ in Equation 4, marked as a black square $(T=0.001 \mathrm{~s})$ in $\boldsymbol{E}$.

waveform, spike waveforms alone, and stereotyped action potential trains in which the actual action potentials are replaced by the average (stereotyped) action potential waveform (Fig. 4D) (see Materials and Methods) (for three tests of the methodology, see Supplement 2, available at www.jneurosci.org as supplemental material). The rate of information transfer for action potential waveforms was $4.2 \pm 1.6$ (mean $\pm \mathrm{SD} ; n=21$ ) times larger than that of the stereotyped impulses. For example, at a spike rate of 10 $\mathrm{Hz}$, the full voltage response carried $800 \mathrm{bits} / \mathrm{s}$, the action potential waveforms $200 \mathrm{bits} / \mathrm{s}$, and the stereotyped impulses $50 \mathrm{bits} / \mathrm{s}$. The high repeatability of both the graded subthreshold activity and of action potential waveforms allowed full voltage responses to have high information transfer rates, comparable with the values measured during naturalistic stimulation in graded potential neurons (Juusola and de Polavieja, 2003). Similarly, the fourfold difference between the true action potential waveforms and stereotyped impulses means that the number of possible messages that can be transmitted using different action potential waveforms is much larger than the number of messages transmissible by spike timing alone. These different messages are distinguishable because of their high reliability, as seen in the repetition experiments or in the small SDs in Figure $1 C$. The main source of unreliability is the spike time jitter, common to codes based on both waveforms and action potential times.

\section{The instantaneous spike rate does not reliably represent different stimulus features}

Experiments using current steps have shown that there is a correlation between spike frequency and spike broadening (Kandel and Spencer, 1961; Aldrich et al., 1979; Fox and Ranck, 1981). We tested whether spike frequency also encodes more dynamic stimulus history. We calculated the average conductance histories preceding different ISIs. Figure $5 A$ shows the distribution of ISIs with different colors identifying 10 groups that we distinguished for analysis. Figure $5 B$ shows the corresponding average conductance histories before the different ISI groups. The average conductances preceding different ISIs resembled those preceding the action potential waveforms in Figure $1 B$, having a transient increase in conductance within $5 \mathrm{~ms}$ before the first spike, and the level of pre-ISI conductance histories followed the same order as the prewaveform conductance histories (compare with Fig. 1B). However, although the waveforms and ISIs encoded on average similar stimulus patterns, the ISIs do not represent these stimulus features well. For the ISI code, the mean conductance histories were less separable and had much larger SDs than with the action potential waveform code, as seen in Figure 5, $C$ and $D$, at 5 and 40 $\mathrm{ms}$ before the first spike, respectively. As in experiments using current steps, spike frequency and spike shape are correlated, but, in our dynamic experiments, the effect is so weak that it is only evident in the average. On a trial-by-trial basis, spike frequency shows a poor correlation to stimulus history compared with the spike shapes.

\section{Encoding into spike shapes in a model of cortical neuron}

The Hodgkin-Huxley model produces action potentials of different shapes that, however, show a rather poor correlation to conductance histories (Supplement 3, available at www.jneurosci. org as supplemental material). Simple models of cortical neurons are known to reproduce the different dynamical behaviors observed in cortical neurons and the spike shape in response to a current step. We use a simple model proposed by Wilson (1999a,b) that includes the spike-producing $\mathrm{Na}^{+}$and $\mathrm{K}^{+}$conductances and the slower $\mathrm{Ca}^{2+}$ and calcium-dependent $\mathrm{K}^{+}$hyperpolarization currents. This simple model already shows some of the relevant characteristics of our experimental results. Figure $6 \mathrm{~A}$ gives the spike height against spike width for the same dynamical stimulation as in our conductance injection experiments (Eq. 1 in Materials and Methods). There is a clear variability in the action potential waveform, here plotted for $37^{\circ} \mathrm{C}$ (Fig. $6 \mathrm{~A}$ ), with a distribution similar to the experimental data. The AMPA average stimulus patterns leading to spikes of different widths (Fig. 6B) show striking similarities to the experimental ones (Fig. $1 B$ ). For every spike waveform, the average conductance pattern has a sudden decrease just before spiking, preceded by an increasing slope. As in the experimental results, wider spikes correspond to higher conductance amplitude. Figure $6 C$ shows the variability of the AMPA conductance pattern for each of the 10 groups of spike waveforms selected at 2.5 and $7.5 \mathrm{~ms}$ before spike production. An encoding of good quality in this simple model has a duration of $10 \mathrm{~ms}$. Action potentials have different waveforms because the underlying conductances have not recovered between spikes. To encode an input history, it is necessary to have slow conductances or slow recovery of predominantly fast voltage-dependent conductances that can act as a memory, in this case of $10 \mathrm{~ms}$ when considering only widths as the only encoding parameter. In the Wilson model, the calcium conductance, with a decay time of 14 $\mathrm{ms}$, can act as the necessary memory for the encoding. Figure $6 \mathrm{D}$ shows the average calcium conductance pattern before each of the 10 groups of spike waveforms selected. These calcium conductance histories are clearly distinguishable up to $10 \mathrm{~ms}$ before spike production and can therefore provide the requisite trace of AMPA receptor input history. Other contributions to spike shape 
1st Extrapolation
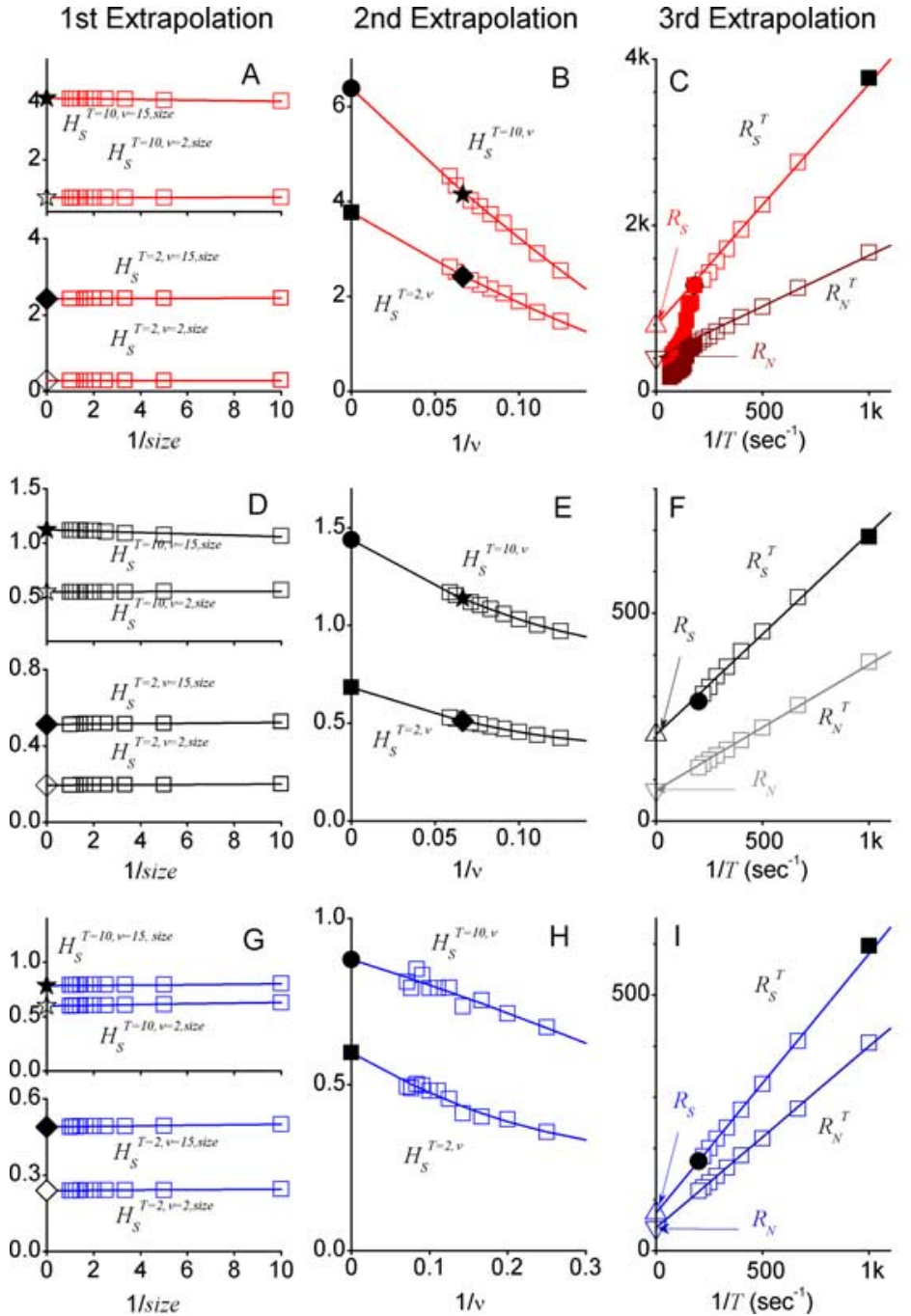

Figure 8. Calculation of the rate of information transfer, Equation 4. A, Extrapolation of the entropy to the limit of infinite size, size $\rightarrow \infty$, for different values of the number of voltage levels and word lengths, shown for $v=2$ and 15 , and $T=2$ letters and 10 letters. $\boldsymbol{B}$, Extrapolation of the entropy to the limit of infinite number of voltage levels, $v \rightarrow \infty$, from the entropies obtained in the extrapolation to infinite size in $\boldsymbol{A}$, here shown for words of $T=2$ letters. $\boldsymbol{C}$, The total and noise information rates, $R_{S}$ and $R_{N}$ in Equation 4, are obtained as the infinite word limit, $T \rightarrow \infty$, of the extrapolated entropies in $\boldsymbol{B}$. For sufficiently long word lengths $T$, the value of the information rate must collapse to zero because of finite data size, here for $1 / T<250$. The extrapolated rate of information transfer for the voltage response, $R=R_{\mathrm{S}}-R_{N}$, is typically $400 \mathrm{bits} / \mathrm{s}$ at a spike rate of $5 \mathrm{~Hz}$. $\boldsymbol{D}-\boldsymbol{F}$, As for $\boldsymbol{A}-\boldsymbol{C}$ but for the spike waveforms, obtained from the voltage response eliminating the variations in the nonspiking intervals of the cell response. Typical information rates for this case are $150 \mathrm{bits} / \mathrm{s}$ at a spike rate of $5 \mathrm{~Hz}$. $\mathbf{G}-\mathbf{I}$, As for $\boldsymbol{D}-\boldsymbol{F}$, but substituting the action potential waveforms by the average waveform. The rate of information transfer for these stereotyped action potentials is typically $30 \mathrm{bits} / \mathrm{s}$ at a spike rate of $5 \mathrm{~Hz}$. Right, Example waveforms of two spikes for each case and a summary of information transfer rate.

might come from the properties of the dendritic tree. An in vivo analysis and a detailed model have shown that dendritic sodium channels contribute to spike shape (Paré et al., 1998). Models using four compartments reproduce this result for the naturalistic stimulation used experimentally and suggest that, at least in the model, this effect is smaller than that of the conductance history (Supplement 3, available at www.jneurosci.org as supplemental material).

\section{Discussion}

Our findings show that input conductance history is encoded in action potential waveforms. This encoding takes place within a single neuron, but different spike waveforms influence synaptic integration and communication with other neurons. Spike waveform thus may function as a 50-ms-long memory of stimulus

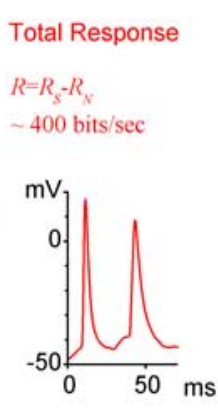

True Spikes

$R=R_{s}-R_{\mathrm{s}}$

$\sim 150 \mathrm{bits} / \mathrm{sec}$

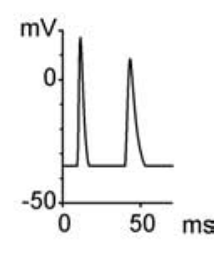

Stereotyped

$R=R_{s}-R_{N}$

$\sim 30 \mathrm{bits} / \mathrm{sec}$

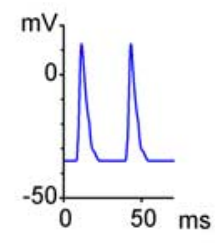

history that can affect the operation of the network. Previous experiments using step currents showed a correlation between spike frequency and spike waveform. Under the more natural stimulation with conductance injection/dynamic clamp (Supplement 1, available at www.jneurosci. org as supplemental material) and using a more naturally structured stimulus made of the sum of unitary synaptic events, we find that spike frequency, although correlated on average to the stimulus history and to spike shape, has a poor correlation on a trial-by-trial basis. For this reason, information rates for action potential waveforms are four times larger than for stereotyped spikes. Because it is now possible to measure conductances in vivo (Wehr and Zador, 2003), our results and the general relationship between conductance history and spike shape found in conductance injection experiments could be further tested.

We also searched for relevant features of the voltage history, finding a correlation between spike height and the preceding voltage averaged over $10 \mathrm{~ms}$, but also between slope to spike and spike threshold, and between threshold and spike height. Thus, both the magnitude and slope of the voltage in the $10 \mathrm{~ms}$ preceding an action potential are related in a systematic way to its shape. At earlier times than $10 \mathrm{~ms}$ before a spike, impact of the slope lessens, especially for wider spikes.

A simple one-compartment model of cortical neuron shows a good correspondence to our experimental results and points to the importance of the dependence of the fast sodium and potassium dynamics on the preceding conductance and of the slow conductances for the encoding of conductance history into waveforms. Models with four compartments show a smaller contribution of the dendritic sodium channels to the variability in spike shape. In general, the distributed nature of synaptic input makes the encoding of conductance input into spike shape dynamically richer and higher in dimension than the case of point conductance injected at the soma, as studied here.

Backpropagating action potentials with different somatic spike shapes differentially shunt incoming excitatory potentials. This affects the next round of integration and thus the communication with postsynaptic neurons even if this communication, because of any normalization of spike shape over long distances in the axon, was to involve spike times or rates only. A different body of work has shown that action potentials of different shapes at the level of the synaptic terminal influence neurotransmitter release (Sabatini and Regehr, 1997; Qian and Saggau, 1999; Stewart and Foehring, 2001), but it remains an open question whether somatic action potentials can directly modulate neurotransmitter release at proximal and dendrodendritic synapses. In addition 
Table 3. The calculations in this study use the following number of points in the extrapolations

\begin{tabular}{|c|c|c|}
\hline & Total entropy & Entropy noise \\
\hline & $H_{s}^{T, v, \text { size }}=H_{s}^{T, v}+H_{S, 1}^{\top, v} /$ size $+H_{S, 2}^{\top, v} / s_{i z e}^{2}$ & $H_{N}^{\top, v, \text { size }}=H_{N}^{\top, v}+H_{N, 1}^{\top, v} /$ size $+H_{N, 2}^{\top, v} /$ size $^{2}$ \\
\hline size $\rightarrow \infty$ & $\begin{array}{l}\text { size being } 1 / 10,2 / 10, \ldots 10 / 10 \text { of data } \\
H_{S}^{T, v}=H_{S}^{T}+H_{S, 1}^{T} / v+H_{S, 2}^{T} / v^{2}\end{array}$ & $\begin{array}{l}\quad \text { size being } 1 / 10,2 / 10, \ldots 10 / 10 \text { of data } \\
H_{N}^{T, v}=H_{N}^{T}+H_{N, 1}^{\top} / v+H_{N, 2}^{\top} / v^{2}\end{array}$ \\
\hline$v \rightarrow \infty$ & $v=8-17(5-15$ for fixed AP waveform $)$ & $v=8-17(5-15$ for fixed AP waveform $)$ \\
\hline & $\begin{array}{l}\text { Mean and } S D \text { of linear extrapolations, } \\
R_{S}^{T}=R_{S}+R_{S, 1} T^{-1}\end{array}$ & $\begin{array}{l}\text { Mean and SD of linear extrapolations, } \\
\mathrm{R}_{\mathrm{N}}^{\mathrm{T}}=\mathrm{R}_{\mathrm{N}}+\mathrm{R}_{\mathrm{N}, 1} \mathrm{~T}^{-1}\end{array}$ \\
\hline $\mathrm{T} \rightarrow \infty$ & calculated using 3-7 linearly aligned points & calculated using 3-7 linearly aligned points \\
\hline
\end{tabular}

AP, Action potential.

to affecting synaptic transmission to postsynaptic cells, spike shape may have a strong effect on spike timing-dependent plasticity of synapses (Markram et al., 1997) and more generally influence second-messenger dynamics (Bi and Poo, 2001).

The participation of stimulus history in the action potential waveform also offers new possibilities for neural computations. Because the precision of input coincidence at the soma controls spike shape (Fig. $1 B$ ), representations of particular coincidences or timing patterns of spatially segregated inputs may be graded rather than discrete, for example representing the number of presynaptic cells activated simultaneously. Furthermore, abundant possibilities for logical gating arise naturally as interactions between forwardpropagating and backpropagating spikes (Larkum et al., 1999) that depend on their shapes.

\section{Appendix: calculation of the rate of information transfer}

Juusola and de Polavieja (2003) have given a practical method to calculate the rate of information transfer for any continuous signal and applied it to photoreceptor voltage responses. A previous, related method developed for discrete signals was given by Strong et al. (1998). This method allows the calculation of the rate of information transmission of action potentials as graded signals. Supplement 2 (available at www.jneurosci.org as supplemental material) gives three tests of the methodology for finite data sets. In the following, we first give the information-theoretic quantities of interest and then discuss how to calculate them using finite data sets. The Shannon (1948) theory of communication is built around the notion of statistical dependence between input and output. In our case, input and output are the variations of total synaptic conductance or the current, $C$, in an interval $T$ and the changes in voltage signal, $S$, in the soma in the same interval. The statistical information between the variations of synaptic conductance, $C$, to the output voltage response of the cell, $S$, is contained in their joint probability distribution, $P_{C S}$. If the output is independent of the input, then their joint probability is the product of their individual probabilities, $P_{C S}^{\text {indep }}=P_{C} P_{S}$. The mutual information, $I_{C S}$, measures the statistical dependence by the distance to the independent situation, given by

$$
I_{C S}=\sum_{i, j} P_{C S}\left(c_{i}, s_{j}\right) \log _{2}\left(\frac{P_{C S}\left(c_{i}, s_{j}\right)}{P_{C}\left(c_{i}\right) P_{S}\left(s_{j}\right)}\right),
$$

where the indices $i$ and $j$ run over the different synaptic conductance patterns $\{c\}$ and somatic voltage changes $\{s\}$ in the interval $T$, respectively. In the limit of infinite resolution, the sum becomes an integral, or, more generally, when the signal has continuous and discrete components, becomes an integral and a sum. The mutual information can also be rewritten as the difference between the total entropy $H_{S}=-\sum_{i} P_{S}\left(s_{i}\right) \log _{2} P_{S}\left(s_{i}\right)$ and the noise entropy

$$
H_{N}=-\left\langle\sum_{i=1} P_{i}(\tau) \log _{2} P_{i}(\tau)\right\rangle_{\tau}
$$

with $P_{i}(\tau)$ being the probability of finding the $i$ th word at a time $\tau$ after the initiation of the trial across trials of identical stimulation.

We digitize the neural response by dividing the graded response into time intervals $T$ that are subdivided into smaller intervals $t$, where $t=0.5$ $\mathrm{ms}$ is the time resolution of our experiments. This digitization of the response can be understood as containing "words" of length $T$ with $T \mid t$ "letters". The values of the digitized entropies depend on the length of the "words", $T$, the number of voltage levels $v$, and the size of the data file, $H^{T, v, \text { size }}$. Take, for example, the case of the voltage changes in a spiking neuron, given in Figure $7 A$. For only four digitization levels, the voltage changes are represented as in Figure $7 B$. This digitization can be understood in terms of "words" as follows. Say we consider words of two letters, $T=2$, depicted in Figure 7C. Counting the occurrence of these twoletter words in the signal of Figure $7 B$, we find their probabilities of occurrence (Fig. 7D). A naive calculation of the total entropy would use this distribution and obtain the value marked as a black square in Figure 7E. A similar naive calculation for the noise entropy uses repeated trials, as in Figure 7F. From these trials, we calculate the distributions at different times, $P_{i}(\tau)$, two of them shown in Figure 7, $G$ and $H$. The corresponding naive noise entropy is given in Figure $7 E$ as a red circle. The rate of information transfer can be obtained from the limits of infinite word length $T$, infinite number of voltage levels $v$, and infinite size of the data file as the difference between the total entropy, $R_{S}$, and noise entropy, $R_{N}$, rates:

$$
R=R_{S}-R_{N}=\lim _{T \rightarrow \infty} \frac{1}{T} \lim _{v \rightarrow \infty \text { size } \rightarrow \infty} \lim \left(H_{S}^{T, v, \text { size }}-H_{N}^{T, v, \text { size }}\right) .
$$

The problem for practical calculations is to obtain these limits. Our approach is to extrapolate the values of the naive entropies to the limits in Equation 4. Take, for example, the case of the calculation of the infinite word size limit for the total and noise information rates in Figure $7 E$. For the highest word lengths considered, the values obtained deviate from the general trend, and the final information rate tends to zero because of the lack of data. When the amount of data are sufficient, the calculation at different word lengths shows a trend that we can extrapolate to the infinite word length limit. The calculation of the rate in Equation 4 involves three concatenated extrapolations that are under control when the linear term dominates in the extrapolation. Figure 8 gives the three extrapolations needed for the cases considered in the main text: the complete voltage waveform, the action potentials, with their waveforms intact but eliminating the nonspiking voltage changes, and the stereotyped action potentials made by further substituting the actual potential waveforms by the average waveform (Table 3 ).

To summarize, the triple extrapolation method uses the original expression for the rate of information transfer without assumptions and makes three concatenated extrapolations to avoid the sampling problem. To control the quality of the extrapola- 
tion, we check that the linear term in the extrapolation dominates. Also, we estimate the errors by considering reduced numbers of points in the extrapolations. We have shown previously (Juusola and de Polavieja, 2003) that this triple extrapolation method gives the same results as the Shannon equation for the particular case of Gaussian distributions. The data were analyzed by custom written Matlab software (Juusola and de Polavieja, 2003).

\section{References}

Aldrich RW, Getting PA, Thompson SH (1979) Mechanism of frequencydependent broadening of molluscan neuron soma spikes. J Physiol (Lond) 291:531-544.

Azouz R, Gray CM (2000) Dynamic spike threshold reveals a mechanism for synaptic coincidence detection in cortical neurons in vivo. Proc Natl Acad Sci USA 97:8110-8115.

Barlow HB (1961) The coding of sensory messages. In: Current problems in animal behaviour, Chap XIII (WH Thorpe, OL Zangwill, eds), pp $330-$ 360. Cambridge: Cambridge UP.

Bi GQ, Poo MM (2001) Synaptic modification by correlated activity: Hebb's postulate revisited. Annu Rev Neurosci 24:139-166.

Carandini M, Ferster D (2000) Membrane potential and firing rate in cat primary visual cortex. J Neurosci 20:470-484.

Coates CJ, Bullock AG (1985) Synaptic plasticity in the molluscan peripheral nervous system: physiology and the role of peptides. J Neurosci 42:1233-1250.

Coombs JS, Eccles JC, Fatt P (1955) The electrical properties of the motorneurone membrane. J Physiol (Lond) 130:291-325.

de Polavieja GG (2002) Errors drive the evolution of biological signaling to costly codes. J Theor Biol 214:657-664.

Fox SE, Ranck JBJ (1981) Electrophysiological characteristics of hippocampal complex-spike cells and theta cells. Exp Brain Res 41:399-410.

Geiger JRP, Jonas P (2000) Dynamic control of presynaptic $\mathrm{Ca}^{2+}$ inflow by fast-inactivating $\mathrm{K}^{+}$channels in hippocampal mossy fiber boutons. Neuron 28:927-939.

Gillette R, Gillette MU, Davis WJ (1980) Substrates of command ability in a buccal neuron of Pleurobrabranchaea. I. Mechanisms of action potential broadening. J Neurophysiol 43:669-685.

Grossman Y, Parnas I, Spira ME (1979) Differential conduction block in branches of a bifurcating axon. J Physiol (Lond) 295:283-305.

Haag J, Borst A (1996) Amplification of high-frequency synaptic inputs by active dendritic membrane processes. Nature 379:639-641.

Harsch A, Robinson HPC (2000) Postsynaptic variability of firing in rat cortical neurons: the roles of input synchronization and synaptic NMDA receptor conductance. J Neurosci 20:6181-6192.

Häusser M, Major G, Stuart GJ (2001) Differential shunting of EPSPs by action potentials. Science 291:138-141.

Jackson MB, Konnerth A, Augustine GJ (1991) Action potential broadening and frequency-dependent facilitation of calcium signals in pituitary nerve terminals. Proc Natl Acad Sci USA 88:380-384.
Juusola M, de Polavieja GG (2003) The rate of information transfer of naturalistic stimulation by graded potentials. J Gen Physiol 122:191-206.

Juusola M, French AS (1997) The efficiency of sensory information coding by mechanoreceptor neurons. Neuron 18:959-968.

Kandel E, Spencer WA (1961) Electrophysiology of hippocampal neurons. J Neurophysiol 24:243-260.

Koch C (1999) Biophysics of computation. New York: Oxford UP.

Larkum ME, Zhu JJ, Sakman B (1999) A new cellular mechanism for coupling inputs arriving at different cortical layers. Nature 398:338-341.

MacKay D, McCulloch WS (1952) The limiting information capacity of a neuronal link. Bull Math Biophys 14:127-135.

Mainen ZF, Sejnowski TJ (1995) Reliability of spike timing in neocortical neurons. Science 268:1503-1506.

Markram H, Lubke J, Frotscher M, Sakmann B (1997) Regulation of synaptic efficacy by coincidence of postsynaptic APs and EPSPs. Science 275:213-215.

Paré D, Lang EJ, Detexhe A (1998) Inhibitory control of somatodendritic interactions underlying action potentials in neocortical pyramidal neurons in vivo: intracellular and computational study. Neuroscience 84:377-402.

Qian J, Saggau P (1999) Modulation of transmitter release by action potential duration at the hippocampal CA3-CA1 synapse. J Neurophysiol 81:288-298.

Reinagel P, Reid RC (2000) Temporal coding of visual information in the thalamus. J Neurosci 20:5392-5400.

Robinson HPC (1991) Kinetics of synaptic conductances. Neurosci Res 16:S6.

Robinson HPC, Kawai N (1993) Injection of digitally synthesized synaptic conductance transients to measure the integrative properties of neurons. J Neurosci Methods 49:157-165.

Sabatini BL, Regehr WG (1997) Control of neurotransmitter release by presynaptic waveform at the granule cell to Purkinje cell synapse. J Neurosci 17:3425-3435.

Shannon CE (1948) A mathematical theory of communication. Bell Syst Tech J 27:379-423.

Sharp AA, O’Neil MB, Abbott LF, Marder E (1993) Dynamic clamp: computer generated conductances in real neurons. J Neurophysiol 69:992-995.

Stewart AE, Foehring RC (2001) Effects of spike parameters and neuromodulators on action potential waveform-induced calcium entry into pyramidal neurons. J Neurophysiol 85:1412-1423.

Strong SP, Koberle RR, van Steveninck RRD, Bialek W (1998) Entropy and information in neural spike trains. Phys Rev Lett 80:197-200.

van Steveninck RRD, Laughlin SB (1996) The rate of information transfer at graded-potential synapses. Nature 379:642-645.

Wang LY, Kaczmarek LK (1998) High-frequency firing help replenish the readily releasable pool of synaptic vesicles. Nature 394:381-388.

Wehr M, Zador A (2003) Balanced inhibition underlies tuning and sharpens spike timing in auditory cortex. Nature 426:442-446.

Wilson HR (1999a) Simplified dynamics of human and mammalian neocortical neurons. J Theor Biol 200:375-388.

Wilson HR (1999b) Spikes, decisions and action: the dynamical foundations of neuroscience. New York: Oxford UP. 